\title{
New self-incubation IVF not yet cheaper
}

$\mathrm{H}$ opes that a new invitro fertilization (IVF) alternative recently approved in Canada could cut the price of IVF in half are so far unfounded. The only Canadian clinic that offers the selfincubation service charges more for it, not less.

The INVOcell device, approved by Health Canada earlier this year, has been touted as a potentially cheaper IVF option. Jason Broome, CEO of the Canadian Fertility Institute, which holds the Canadian rights to INVOcell, and fertility doctor Kevin Doody, co-head of the Center for Assisted Reproduction in Bedford, Texas, recently demonstrated that a streamlined protocol using the device could be just as effective as traditional IVF but at a much-reduced cost.

The new approach involves scaling down all parts of the process. In traditional IVF, a woman is given drugs to make her ovaries produce extra eggs. She is then monitored every few days using ultrasound and hormone blood tests, and the drug dosage is adjusted. To keep costs down, Doody prescribed the drugs, conservatively, based only on body weight and anti-Müllerian hormone testing, which estimates egg supply. He did a single monitoring ultrasound on day 10, and based on those results, scheduled the egg harvest for day 12,13 or 14 . Retrievals and transfers were timed not to fall on weekends, to further control costs.

Rather than putting eggs and sperm together into a lab-controlled incubator, as with traditional IVF, he allowed the gametes to mingle for an hour or so, then placed the as-yet unfertilized eggs, with shortlisted sperm clinging to the outer shell, along with culture medium, into a device known as INVOcell. This was placed inside the vagina, secured by a diaphragm, and left in place for five days.

Doody presented preliminary results of a study of the new process at the October meeting of the American Society for Reproductive Medicine. The trial sample was small; half the 40 women received traditional IVF and half underwent the new process. Rates of fertilization, pregnancy and live birth were similar in both groups, but the INVOcell process was half the cost.

The cylindrical, plastic INVOcell device measures $4 \mathrm{~cm}$ by $3 \mathrm{~cm}$. It has an outer chamber to protect against contamination, but is permeable to gas. The mother's own body maintains optimal levels of oxygen and carbon dioxide, keeps the temperature steady and acts as a filter. "We're using, essentially, the intended-mom's kidneys and liver to filter out environmental toxins," says Doody.

The cost-savings of not using a sophisticated lab, with 24-hour monitoring and quality control, is enormous, Doody says. Costs were further reduced by not needing weekend staff and using fewer drugs. He says he expects to offer the scaled-down treatment for US\$6000, half of what he charges for regular IVF.

Broome says he'd like to use INVOcell to make assisted reproduction more financially accessible to Canadian couples. "Lots of patients don't proceed with IVF because of cost." IVF can cost upward of $\$ 10000$ per attempt in Can- ada, where, outside Quebec, it is typically not publicly funded. The INVOcell device costs about $\$ 450$ in Canada.

Both Broome and Doody say they believe INVOcell could make fertility treatment more geographically accessible, too, by bringing it to smaller centres that don't have access to stateof-the-art fertility labs.

However, even some Canadian INVOcell enthusiasts doubt the device will change much in terms of cost and access. John McNaught, a fertility doctor in London, Ontario, who used INVOcell in an as-yet unpublished trial conducted in 2013, says the real costs are not due to the lab but to the surgical centre required to do egg retrieval under anesthetic. The only cost savings, he predicts, will come from using fewer drugs. "It's not going to differ in price as much as we'd hoped," he says.

Robert Casper, medical director of the Toronto Centre for Advanced Reproductive Technology (TCART), agrees. "You still need to have a good set up for egg retrieval, and you still need an embryologist," he says. Currently, TCART is the only clinic in Canada offering INVOcell as a service. Casper considers it an "add-on" to regular IVF, and charges an additional \$500 for it. He points out that some women actually prefer to carry their own embryos.

Roger Pierson, a professor of Obstetrics and Gynecology at the University of Saskatchewan, who specializes in assisted reproduction, agrees that INVOcell isn't going to make IVF affordable to all, or turn every little Canadian town into a fertility centre. But he calls it "a good step forward" from "an excellent lab with outstanding science." — Alison Motluk, Toronto, Ont.

CMAJ 2015. DOI:10.1503/cmaj.109-4949 